\title{
Cepheids in IC 4182, Calibration of SNIa 1937C and the Hubble constant
}

\author{
A. Saha ${ }^{1}$, A. Sandage ${ }^{2}$, N. Panagia ${ }^{1}$, G. A. Tammann ${ }^{3}$, L. \\ Labhardt $^{3}$, H. Schwengeler ${ }^{3}$, F. D. Macchetto ${ }^{1}$ \\ ${ }^{1}$ Space Telescope Science Institute, Baltimore, MD, USA, ${ }^{2}$ Observatories of the \\ Carnegie Institution of Washington, Pasadena, CA, USA, ${ }^{3}$ Universitat Basel, \\ Basel, Switzerland
}

\begin{abstract}
Observations with the Wide Field Camera on HST were examined, and 27 Cepheids were discovered. Photometry and period analysis have produced unambiguous light curves free of alias. The observed P-L relation has a slope consistent with seminal calibration studies of Galactic and LMC Cepheids. An apparent distance modulus (in V) of $28.47 \pm 0.10$ to IC 4182 is derived. This implies that $M_{V}(\max )$ for SNIa $1937 \mathrm{C}$ is $-19.92 \pm 0.2 \mathrm{mag}$, independent of everything except differential absorption between the Cepheids and the supernova. Using this to calibrate Hubble diagrams for SNIa by several authors, and allowing for a 1- $\sigma$ uncertainty in the absolute magnitude from the calibration of only one SNIa, we obtain $H_{0}=45 \pm 14 \mathrm{kms}^{-1} \mathrm{Mpc}^{-1}$.
\end{abstract}

\section{Introduction}

IC 4182 is a nearby Sdm galaxy which produced a type Ia supernova (SNIa) $1937 \mathrm{C}$ (Baade and Zwicky 1938). Hubble diagrams constructed from data on accurately measured SNIa in more distant galaxies $\left(v>1600 \mathrm{kms}^{-1}\right)$ indicate that the dispersion in the peak brightness of SNIa disperse about a mean value by $0.5 \mathrm{mag}(1-\sigma)$. Thus they are reliable standard candles once the absolute peak magnitudes of a few such SNIa are measured. Here we describe the work to determine the distance to IC 4182 through the discovery and photometry of Cepheids using the Hubble Space Telescope (HST), and thus the calibration of the absolute peak magnitude of SNIa 1937C.

\section{Data and Photometry}

A field in IC 4182 was imaged at 20 different epochs by the Wide Field Camera (WFC) on the HST in the F555W bandpass (almost equivalent to $V$ ). The total time spanned by these observations was 47 days, and the epochs were judiciously spaced in time by amounts varying from 15 hours to 6 days to eliminate aliassing over the period range 3 to 45 days. At each epoch, two back to back exposures were made, each 2100s long. Each such exposure pair was checked for exact registration. To remove the cosmic rays, anti-coincident pixels (at the 6- $\sigma$ level) were flagged, and 
the lower of the two values for a given pixel were retained in both the sub-exposures. These were then co-added to a single image, thus yielding 20 images at the 20 epochs that are cleaned of cosmic rays.

A modified version of the DoPHOT (Mateo \& Schechter 1989) point spread function (PSF) fitting program was run to perform star identification and photometry. In this version an analytic PSF model is fit to the central cores of the stars. The reported brightness measures the rise of the central spiked core (of the spherically aberrated image) of a star relative to its own 'wings'. This procedure preserves the relative magnitude difference from one star to another, without having to ascertain the 'true' background under each object. For faint objects this procedure is tantamount to a maximum S/N extraction. The analytic PSF has fewer procedural problems for fitting to the severely undersampled data. The goodness of fit against the model stellar PSF is used to eliminate non-stellar objects and other image pathologies: in particular low level cosmic rays that are not trapped by the anti-coincidence method described above. Pathological pixels are 'turned off' and not used in any further computation. This procedure is crucial to getting good relative magnitudes. The final result is a list of bona-fide stars with positions and relative magnitudes with associated individual error estimates. At this point these magnitudes have arbitrary zero-points that vary from chip to chip and exposure to exposure: they are only self-consistent within any chip on any single epoch, and even then there is variation of order 5 percent (from control experiments on HST Science verification data on NGC 1855 that were offset in position and telescope roll angle) depending on position, since even the PSF of the cores of stellar images varies with position.

A 'template' image was constructed for each chip by stacking several exposures (using only integer pixel shifts to adjust the registration), and the PSF fitting process was applied to it as well, resulting in a more comprehensive list of stars and their relative magnitudes. We shall call the relative magnitudes obtained on the template image the instrumental magnitude. Bear in mind that the instrumental magnitudes thus defined are independent from chip to chip, and one must determine the zeropoint to go to true magnitudes separately for each chip. For each of the four chips, the stars (from the DoPHOT generated lists) from each epoch were matched by position to those on the template. By examining the difference in relative magnitudes of the stars in any epoch against the instrumental magnitudes of the same stars on the template, the shift needed to go from the relative to instrumental magnitude can be determined and applied. Since this is based on the ensemble average for hundreds or thousands of objects, the shift is extremely robust and precise, to better than 0.02 mag. Note that the error in transforming from relative to instrumental magnitudes is much less than the uncertainty in magnitudes due to the PSF variation across each chip, since the observations were all made in the same position and orientation. For a given star, the relative systematic error in the instrumental magnitudes from one epoch to another is only $0.02 \mathrm{mag}$, although its magnitude relative to other stars in 
the chip may be uncertain by 0.05 mag.
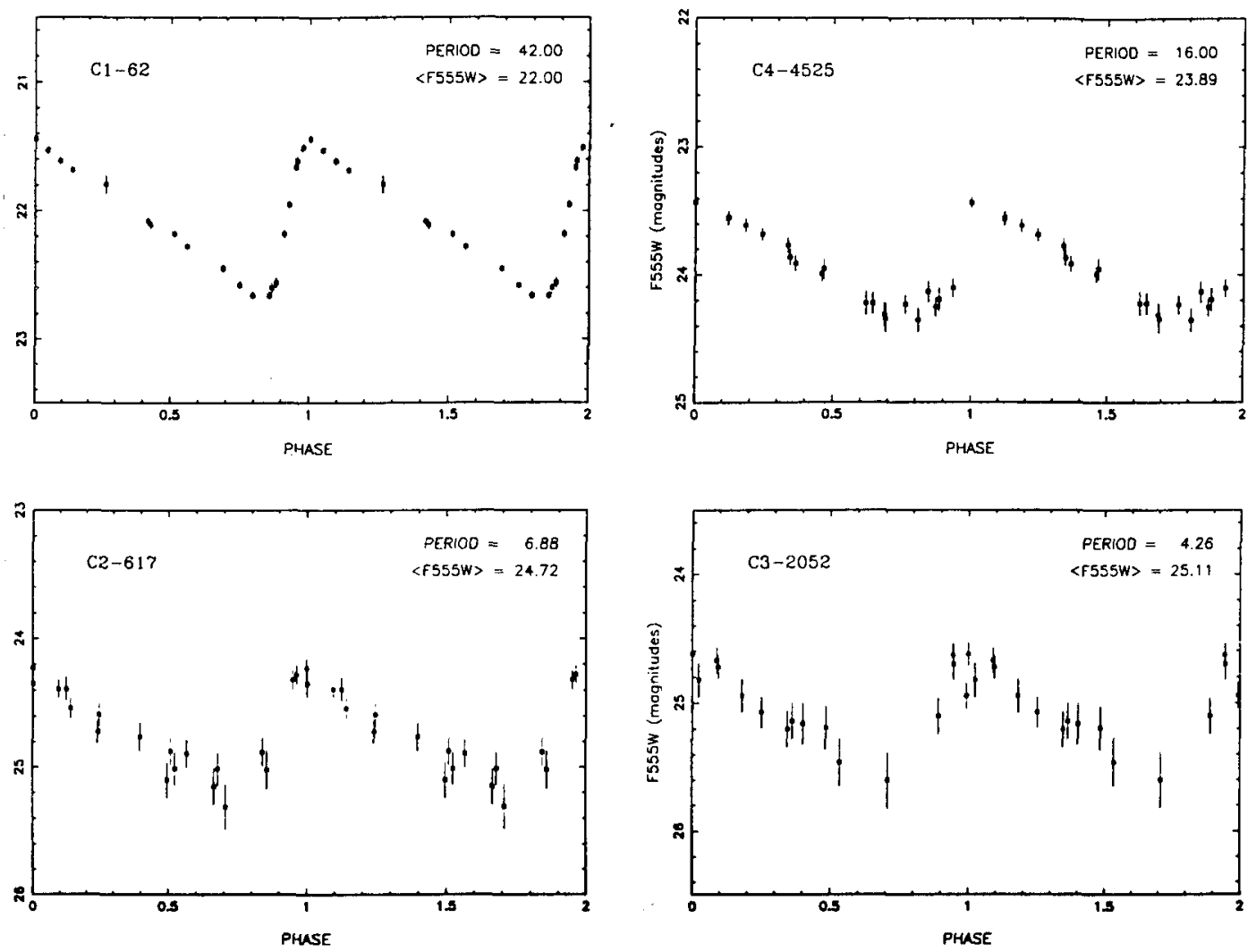

Figure 1. Representative light curves of Cepheids found in IC 4182.

The instrumental magnitudes, as operationally defined above, differ from the 'true' magnitudes by an additive constant, $C$, which is separate for each chip and must be evaluated independently. To do so, several stars in each chip that are relatively bright and apparently isolated were chosen, and the instrument Data Number (DN) counts within a 30 pixel radius aperture were determined. Using the calibration 
given for DN to magnitudes in the F555W system in Faber et al. 1992 (Table 12.15), the value of $C$ (as above) for each chip was derived. In the more isolated areas the internal consistency (star to star variations within the chip area) in $C$ was found to be within the 0.05 mag expected scatter. In the most crowded areas this concordance is hard to come by, and the uncertainty in the determination of $C$ can be as much as $0.1 \mathrm{mag}$. Once determined, $C$ can be applied to each object in each epoch to obtain all magnitudes on the standard F555W system.

The search for variables was made using a $\chi^{2}$ test, followed by a 'periodicity' test based on a variant of the Lafler and Kinman (1965) algorithm. This technique has been demonstrated in Saha and Hoessel (1990). Unambiguous Cepheid light curves were obtained for 27 of the detected variables, ranging in period from 3 to 45 days. The special timing scheme applied in the observations assured the absence of period aliassing in this range. Fig. 1 shows 4 representative light curves, arranged in order of period. Note how as one goes fainter, the error bars increase.

The mean magnitudes in F555W are plotted against $\log P$ in Fig. 2, which is the familiar P-L relation. We consider the Sandage and Tammann (1968) P-L relation which gives:

$$
<M_{V}>=-2.83 \log P-1.37
$$

Comparison with the Feast and Walker (1987) and Madore and Freedman (1991) P-L realtions for $M_{V}$ shows that all three agree to within 0.1 mag over the period range 3 to 40 days. The continuous line in Fig. 2 shows the fit of the Sandage and Tammann (1968) slope to the data for IC 4182, and the dash-dot lines on either side mark the intrinsic scatter in the P-L relation due to the finitc width of the instability strip in temperature. Note that the fainter Cepheids appear to be systematically too bright compared to the fitted mean ridge-line. We attribute this to the bias arising from the incompleteness of discovering Cepheids at the very faint limit. The fit is done to all the data irrespective of the bias, which has the effect of deriving a slightly smaller distance modulus. The fitted equation is:

$$
<F 555 W>=-2.83 \log P+27.16
$$

The formal difference between $F 555 \mathrm{~W}$ and $V$ magnitudes is documented in Harris et al. (1991), and the difference is about 0.03 mag. For the present we make no distinction between the two, implying an apparent distance modulus $(m-M)_{a p p}=$ 28.53. Following the same procedure, but using the Feast and Walker (1987) and the Freedman and Madore (1991) calibrations yields $(m-M)_{a p p}=28.43$ and 28.45 
respectively. We adopt $(m-M)_{a p p}=28.47 \pm 0.10$

\section{$P-L$ relation}

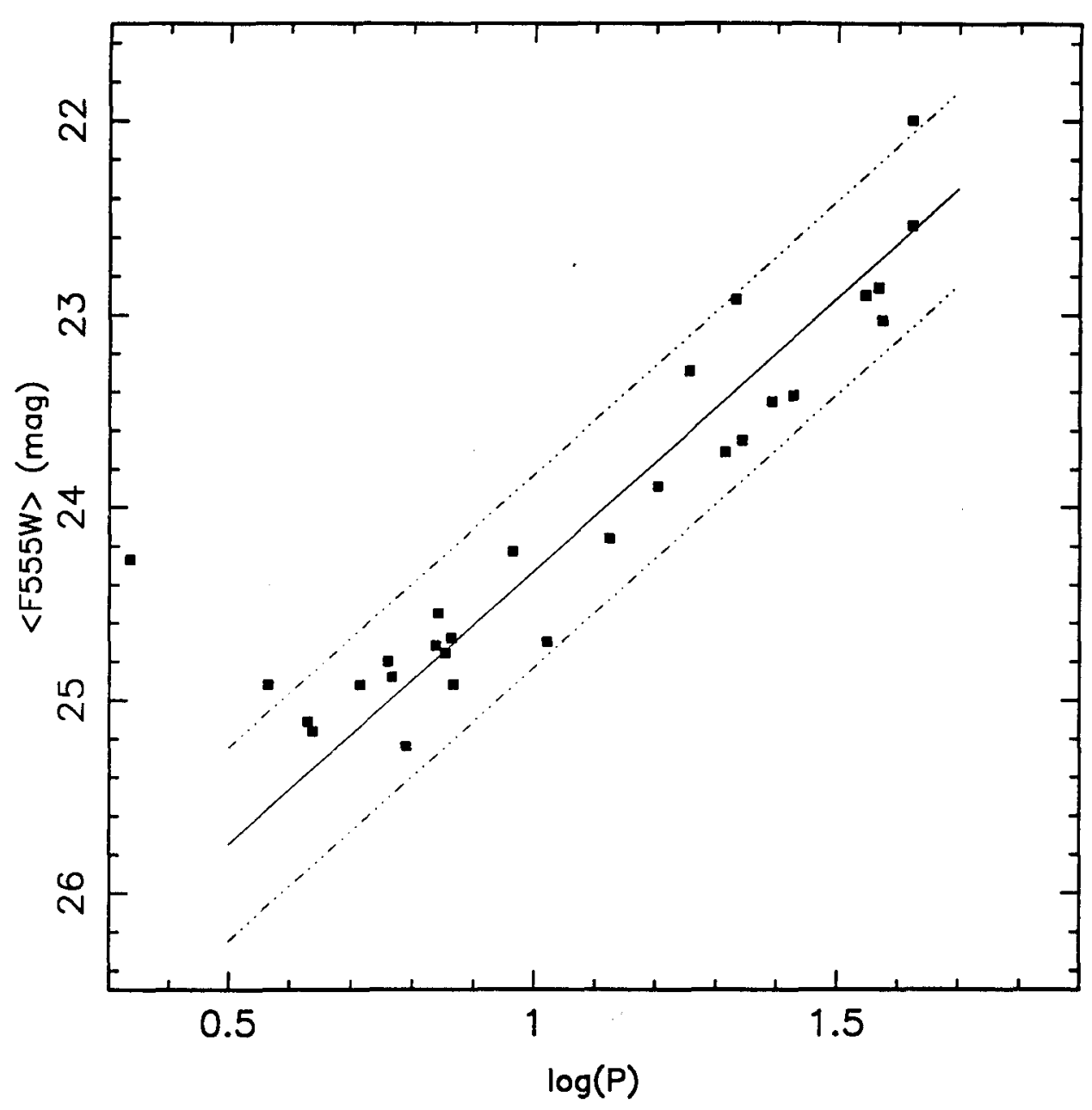

Figure 2 The P-L relation for Cepheids in IC 4182.

3. The Peak brightness of $\mathrm{SN} 1937 \mathrm{C}$ and the value of $H_{0}$.

The photographic $m_{p g}$ light curve of SN $1937 \mathrm{C}$ were well determined by Baade and Zwicky (1938). The $V$ light curve was determined by Beyer (1939). Standard template light curve fitting (Leibundgut et al. 1991) gives $V_{\text {max }}=8.55$ and $M_{p g}(\max )$ 
$=8.50$ for this type Ia supernova. Since our apparent modulus to IC 4182 is determined in $V, M_{V}(\max )=19.92 \pm 0.2 \mathrm{mag}$, as long as there is no differential extinction between the Cepheids and the location of SN $1937 \mathrm{C}$.

We see already in Fig. 2 that the Cepheids essentially all lie within the range of the intrinsic scatter, indicating that the differential absorption is less than $0.4 \mathrm{mag}$ in our field, which includes the location of SN 1937C. Further, from photometry on the coadded images, it is seen that resolution of the brightest 'normal' giants occurs at $F 555 W=25.8 \mathrm{mag}$. IC 4182 lies in the direction of the North Galactic Pole, and is thus free of extinction from our own galaxy. Since it is face on, the brightest 'normal' giants seen should be the ones on the closer side of the 'disk', and so free from internal extinction within IC 4182. The giant branch termination point has $M_{V} \approx-2.5$, thus implying a distance modulus of 28.3 , which, as argued above, is free of the extinctions within IC 4182. This value agrees very well with the apparent modulus of 28.47 derived from the Cepheids, thus showing that there is no significant internal extinction to be accounted for, let alone differential extinction (the latter is the only factor that affects our determination of $M_{V}$ for SN 1937C).

We apply the result obtained above to calibrate the SNIa Hubble diagram. Consider first the one given by Tammann and Leibundgut (1990), for all types of galaxies, where they obtain:

$$
M_{B}(\max )=-19.63( \pm 0.09)+5 \log \left(H_{0} / 50\right)
$$

and a mean value of the color at maximum of $(B-V)_{0}=-0.27$, which can be used to transform the above relation to $M_{V}(\max )$ :

$$
M_{V}(\max )=-19.36 \pm 0.09+5 \log \left(H_{0} / 50\right)
$$

Similarly, from Della Valle and Panagia (1992) we obtain (using the appropriate $(B-V)_{0}=-0.16$ from their particular sample) we obtain:

$$
M_{V}(\max )=-19.96 \pm 0.18+5 \log \left(H_{0} / 50\right)
$$

Again, from Sandage and Tammann (1982), who had a sample of SNIa in E and S0 galaxies only (i.e. no internal extinction), using again $(B-V)_{0}=-.27$, we obtain:

$$
M_{V}(\max )=-19.74( \pm 0.24)+5 \log \left(H_{0} / 50\right)
$$

The above values bracket other mean calibrations in the literature.

If $\mathrm{SN} 1937 \mathrm{C}$ is a mean type Ia supernova, the above calibrations can be used to give a range for $H_{0}$ :

$$
39 \pm 5 \leq H_{0} \leq 51 \pm 7 k m s^{-1} M_{p c}^{-1}
$$

The dominant uncertainty in this result arises from the fact that SN 1937C may not itself lie on the mean line of the Hubble diagram. The Tammann and Leibungut (1990) and Della Valle and Panagia (1992) Hubble diagrams show an intrinsic scatter 
in the $B$ magnitudes of $0.50 \mathrm{mag}, 1-\sigma$. This implies a $24 \%$ uncertainty in the determination of $H_{0}$. The conservative estimate from the determination of the absolute magnitude of this one supernova is thus:

$$
H_{0}=45 \pm 14 \mathrm{kms}^{-1} \mathrm{Mpc}-1 .
$$

The calibration of other SNIa in the above manner will significantly reduce the uncertainty.

\section{References:}

Baade W., Zwicky, F., 1938, Astrophys. J., 88, 418.

Beyer. M. 1939, Astron. Nachr., 268, 341.

Della Valle, M., Panagia, N. 1992, Astron..J. 104, 696.

Faber, S. et al. 1992, Wide Field/Planetary Camera Final Orbital Science Verification Report, STScI.

Feast, M., Walker, A. R., 1987, ARA\&A, 25, 345.

Harris, H. C., Baum, W. A., Hunter, D. A., Kreidl, T. J. 1991, Astron. J. 101, 677.

Lafler, J., Kinman, T. D., 1965, Astrophys. J. Supp., 11, 216.

Leibundgut, B., Tammann, G. A., Cadonau, R., Cerrito, D., 1989, Astron. \& Astrophys. $\mathbf{8 9}, 537$.

Madore, B., Freedman, W. L., Pub. Astron. Soc. Pac., 1991, 103, 933.

Mateo, M., Schechter, P. 1989, in The First ESO/ST-ECF Workshop on Data Analysis, ed. P. J. Grosbol, F. Murtagh, and R.H. Warmels (ESO, Munich), p. 69.

Saha, A., Hoessel, J. G., 1990, Astron. J., 99, 97.

Sandage, A., Tammann, G. A., 1968, Astrophys. J., 151, 531.

Sandage, A., Tammann, G. A., 1982, Astrophys. J., 256, 339.

Tammann, G. A., Leibundgut, B., 1990, Astron. \& Astroph., 236, 9.

\section{DISCUSSION}

E. BÖHM-VITENSE: I would like to suggest that the systematic deviations that you find for your period-magnitude relation are due to a change from fundamental mode pulsation to first overtone pulsation for periods of about 10 days as is expected theoretically.

A. SAHA: We had ascribed the deviations to incompleteness, but it would be worthwhile to test if applying a period shift to account for change to first overtone would reduce the deviations.

W. FREEDMAN: A comment on your statement that the data for IC 4182 are confined to within the ridge lines of the PL relation and therefore suffer low differential extinction. It is also the case that the PL relations for the Cepheids in the inner regions of M31 do not exhibit a large dispersion and yet suffer up to 1 magnitude $B$ absorption in the mean. In any case you do have $I$ data that will allow you to determine the extinction of the IC 4182 Cepheids directly.

A. SAHA: Yes, but the calibration of the brightness of SN1937C depends only on the differential extinction, although the true distance modulus of IC 4182 is extinction dependent. We will have better limits on the value of the absolute extinction, when we've analysed the $I$ data, as you've said. 
M. PIERCE: I'm a bit concerned that there are internal inconsistencies in your determination of $M_{B}(\max )$ for $\mathrm{SN} 1937 \mathrm{C}$. If you take the values of $m_{V}(\max )$ and $M_{p g}(\max )$ you gave in your talk and the canonical correction for $p g$ to $B$ mags along with the intrinsic color of $(B-V)_{\max }=-0.25$ you get $E(B-V) \approx 0.43$ and $A_{B} \approx 1.4 \mathrm{mag}$. The result is that $H_{0} \approx 18 \mathrm{kms}^{-1} M \mathrm{Mc}^{-1}$, a ridiculously small value. Could you comment on the importance of reddening and extinction.

A. SAHA: Your argument would be valid if one could assert that the observed magnitudes and colors of SN1937C as well as the intrinsic color of SNIa's have negligible associated errors and/or uncertainties. When you take your inferred $E(B-V)$ and derive $A_{B}$, you have multiplied any measurement errors also by a factor of 4 . Thus an entirely plausible error in measurement of the color of SN1937C of 0.2 mag say, plus an uncertainty in our knowledge of the intrinsic color at maximum of SNIa's by a like amount translates to an error that is 4 times as large, i.e. larger than 1 mag. Therefore $I$ think it is misleading to follow your line of argument, since it can lead to fictitious results based solely on amplifying the errors and uncertainties by a factor of 4 . The procedure we have chosen to follow, transforms the Hubble diagram obtained in $B$ to one in $V$ using the intrinsic colors and extinctions derived self consistently from within that data-set. This Hubble diagram in $\mathrm{V}$ is then compared against the $V(\max )$ value of $1937 \mathrm{C}$ obtained from Beyer's data. This procedure avoids the problem of a $4 \times$ artificial magnification of errors. 